\section{J.G. Ozbolt}

School of Nursing and Division of Clinical Informatics, Department of Health Evaluation Sciences, School of Medicine, The University of Virginia, Charlottesville VA, USA.

\section{Introduction}

Computer-based systems can play a number of roles in facilitating the clinical management of patients and in managing health information. At one extreme are systems that operate as medical devices, monitoring patients and sounding alarms or adjusting treatments as necessary. At the opposite extreme are systems that capture limited information about patients that can be retrieved for consideration by clinicians or researchers. Between these extremes are multi-functional, interactive systems for supporting clinicians in patient care. Across the spectrum, each type of system must be evaluated interms of the reliability of the data, the accuracy of the algorithms by which it processes the data, its success in performing its intended functions, and the "value added" or perceived usefulness itcontributes to the clinical or research endeavor compared to alternative approaches. One or another of these aspects may be emphasized in a particular evaluation, but all must be considered for a comprehensive assessment of the contributions of these systems to health care and the health of populations.

The papers in this section provide examples of systems across the specfrum. Muiller et al. [1] investigated the

\title{
Synopsis
}

\section{Health and Clinical Management}

feasibility of machine learning as a means of generating production rules for an intelligent ventilator alarm system. Short et al. [2] conducted a clinical trial of the control of anesthesia infusion by a computer program based on a pharmacokinetic model. At the other end of the spectrum from these studies of medical devices are two investigations of information technologies for capturing and retrieving patient information, in the one instance for the registration and disposition of victims of mass casualties [3], and in the other instance for large-scale follow-up of the outcomes of primary prevention interventions [4]. Between these extremes are two contrasting reports of experiences with clinical documentation systems. Rotman etal. [5] described the lessons learned from a failed implementation of a computerbased physician workstation that had been designed to facilitate and improve ordering of medications. Those lessons are not identical to, but are consistent with, the recommendations of Leiner and Haux [6] in their protocol for systematic planning and execution of documentation projects.

As these papers demonstrate, the range and diversity of challenges to informatics in health and clinical management are very great. As the papers also demonstrate, however, investiga- tors are rising to the challenges, and the quality of solutions can be expected to increase.

\section{Building Intelligent Medical Devices}

Müller etal. [1] investigated whether acombination of modeling, simulation, and machine learning could serve as an effective and efficient alternative to eliciting knowledge from experts for building intelligent alarm systems for ventilator therapy. The investigators developed a mathematical model of relationships among airway pressure, expiratory gas flow, and $\mathrm{CO}_{2}$ concentration at the endotracheal tube during volume-controlled ventilation with constant ventilator settings, for both normal functioning of the breathing circuit and for breathing circuit mishaps (leaks and obstructions). They also created 94 physiologically different simulated "patients" by varying airway resistance and lung/thorax compliance values. Using the mathematical model, the investigators calculated and simulated signal values for pressure, flow, and $\mathrm{CO}_{2}$ under normal conditions and different fault conditions for all modeled patients. The calculated signal values were validated by comparison with values obtained from ventilated 
sheep. Although some differences were noted, the investigators characterized these as "marginal."

Differences in respiratory signals among the normal and the various fault situations were the basis for machine learning. A pattern recognition algorithm was used to identify the combination of signal parameters associated with each normal or fault condition, the condition being identified a priori. A training set was created from 54 unique simulated patients. From data on simulated breaths of these patients, the machine-learning algorithm created rules linking signal values to breathing circuit events. These rules were tested by challenging them to classify events in the remaining 40 simulated patients. The rules reached 99\% accuracy in this classification task. The rules reached $100 \%$ accuracy in classifying events from actual measurements taken at a lung simulator.

Thus, machine learning made it possible to generate production rules automatically, saving time and increasing consistency over eliciting knowledge from experts. The results demonstrated the feasibility of developing an automatically generated alarm system based on a relatively simple mathematical model.

Can a data-based model of pharmacokinetics adequately control the administration of anesthesia? In this study, Short et al. [2] programmed a model developed previously by other investigators to control infusion of propofol in a sample of adult Asian patients in a Hong Kong hospital. The delivery system was programmed to achieve rapidly and then to maintain a constant concentration of medication in the arterial blood. Predicted concentration was compared to measured concentration in arterial blood samples taken at 5 to 10 minute intervals during infusion and at 15 to 20 minute intervals after the cessation of infusion. The system was found to be better at maintaining a constant concentration than at predicting what that concentration would be. For satisfactory clinical performance, it was necessary to adjust the target concentration for an individual on the basis of clinical response. Moreover, the model described the infusion phase of anesthesia more accurately than the recovery phase. Overall, the model performed better for this sample of healthy adults with a tightly defined range of age and degree of obesity than in a sample of pediatric patients in the same hospital.

\section{Technologies for Data Capture and Retrieval}

To improve the accuracy, completeness, and speed of acquisition of patient information during mass casualty incidents, the Emergency Hospital of Utrecht, The Netherlands, uses a computer-based system with barcoded identifiers for patients, injuries, facilities, and locations. In evaluation studies [3], the system compared favorably with handwritten records, resulting in fewer inaccuracies. Admitting personnel were able to use the system without extensive training. Physicians, nurses, and logistic personnel reported that information available through the system enabled them to provide better patient care and management. The system communicates with the overall hospital information system, giving rise to concerns for the security of information. Safeguards have been implemented, with policy decisions favoring the common good over potential abuse.

The West of Scotland Coronary Prevention Study Group (WOSCOPS) compared patient self-report of adverse coronary events to searches of databases held in the Scottish Record Linkage System (SRL) in detecting myocardial infarction or heart disease death in a randomized double-blind clinical trial of pravastatin versus placebo in the primary prevention of coro- nary heart disease [4]. Events identified by computerized record linkage were compared with those reported by. patients. Discrepancies were followed up by contacting the physician and scrutinizing the individual patient's records.

In a prospective epidemiological study involving a sample of 80,184 men, there was $100 \%$ agreement in records of deaths. Each system recorded some adverse events that were not recorded in the other system because of linkage error or failure to capture the data. In all, WOSCOPS identified $89 \%$ of events and SRL identified $95 \%$.

Thus, although neither system was perfect, the two approaches yielded cross-validation that both were reasonably accurate. The investigators concluded that, especially in community-based studies of primary prevention where follow-up was difficult, computerized record linkage "provides not only significant cost advantages. but also potentially more complete follow-up of serious adverse events" [4, p. 1448]. The investigators noted, however, the ethical issues in gaining. access to patient records. They argued that linking subjects' records to national databases "in order to obtain. output in the form of summary statistics, does not breach any rules of confidentiality and is comparable with routine uses for such data" [4, p. 1449].

\section{Implementing Clinical Documentation Systems}

Rotman et al. [5] designed a ran domized controlled trial of a physician workstation to avoid the confounding effects of self-selection of users and of extraneous events that might affec the outcome variables. To increase statistical power, they used a two period parallel design. Unfortunately the physicians randomly selected to tri the new workstation did not use 
enough to influence clinical or economic outcomes. User satisfaction declined in the workstation group and increased in the control group. The most valuable aspect of the study is the lessons learned from the failed trial.

It is important to note that all physicians were in the habit of using an existing computer-based system. The old system continued to be available to all, but the new system was available only to those in the intervention group. The new system offered some features not available in the old system, specifically alerts about drug interactions and about less expensive medications that could be considered as substitutes for medications initially selected by the physician as well as graphical depiction of trends in laboratory results. The old system, however, contained information such as specific laboratory tests and procedures needed by all physicians but not available in the new system. Thus, it was essential for all physicians to continue to use the old system, but optional for those in the intervention group to use the new system. Furthermore, system failures made the new system unavailable in $10 \%$ to $15 \%$ of user sessions. When the system functioned, response time was slow: 30 to 50 seconds to receive laboratory results. During the study period, the old system underwent a number of enhancements, making it more attractive and simultaneously increasing downtime for the new system, which required changes because of its communications with the old system.

These experiences led the investigators to make the following recommendations for studies evaluating information systems:

1. Specify the performance characteristics and support for both the experimental and control systems.

2. Assess the reliability and speed of the computing infrastructure.

3. Investigate what works and does not work with the current clinical workflow and tailor new or modified systems accordingly.

4. Provide sufficient training time to users.

5. Choose an experimental design that maximizes statistical power to detect differences in the relevant outcomes.

6. Design evaluative studies to be informative even if the study does not find positive effects on health or economic outcomes.

a) Distinguish between an intervention that was not used and one that was used but ineffective.

b) Link user feedback to objective measures of system performance.

c) Systematically assess subjective responses of users.

d) Prioritize system improvements.

Following the recommendations of Rotman et al. in conducting evaluation studies would certainly help to understand why a clinical documentation system succeeded or failed. To enhance successes and to avert failures, Leiner and Haux [6] described a protocol for systematic planning and execution of documentation projects that has been used in three projects in a German university hospital. The advantage of this protocol over other approaches is that it begins by asking the purposes for which the documented data will be used. Other steps in the process then follow in logical sequence. The result is better integration of the various aspects and functions of the system and greater assurance that data entered into the system will be retrievable and useful for desired purposes. The authors report anecdotally on pros and cons gleaned from their experience. Many of the cons relate to the disaffection of some organizational members when they learn early in the process that some of their objectives will not be met because of problems of expense or feasibility. Other cons concern the need to reiterate the planning process if fundamental objectives or design decisions change. These negatives, however, are better confronted early and resolved than ignored or deferred. They are in any case outweighed by the pros, which include thorough and integrated planning, improved cos t projections, and enhanced discussions among designers and users. Although for simplicity the examples given relate primarily to documentation by physicians, the protocol is equally applicable to documentation by other disciplines such as nursing and to integrated documentation by all disciplines involved in patient care.

\section{Conclusions}

In building intelligent medical devices, a key challenge is to acquire and to represent the knowledge guiding the algorithm that controls the device. Generating production rules through application of a mathematical model may yield satisfactory clinical performance more efficiently than acquiring knowledge from experts, but careful testing for accuracy, safety, and efficacy is essential. In practice, the device may perform differently from predictions, and adjustments may. be needed for patient safety and clinical effectiveness. New technologies for information capture and retrieval offer benefits in patient management and knowledge discovery but bring risks of loss of privacy and confidentiality. Ethics and public policy as well as technological feasibility must guide the use of such technologies. Finally, the implementation of clinical documentation systems is far more complex than the replacement of one technology with another. Such systems transform work and organizational relationships. If the implementation is to succeed, attention must be given to these transformations and the disruptions they entail. To realize the promise of informatics for health and clinical management, those who develop and pro- 
mote the use of applications must anticipate, evaluate, and accommodate the full range of consequences.

\section{References}

1. Müller B, Hasman A, Blom JA. Building intelligent alarm systems by combining mathematical models and inductive machine learning techniques. Int J Biomed Comput 1996;41:107-24.

2. Short TG, Lim TA, Tam Y. Prospective evaluation of pharmacokinetic model-controlled infusion of propofol in adult patients. Br J Anaesth 1996;76:313-15.

3. Noordergraaf GJ, Bouman JH, van den
Brink EJ, van de Pompe C, Savelkoul TJF. Development of computer-assisted patient control for use in the hospital setting during mass casualty incidents. Am JEmerg Med 1996;14:257-61.

4. The West of Scotland Coronary Prevention Study Group. Computerised record linkage: compared with traditional patient follow-up methods in clinical trials and illustrated in a prospective epidemiological study. J Clin Epidemiol 1995;48:1441-52.

5. Rotman BL, Sullivan AN, McDonald TW, Brown BW, DeSmedt P, Goodnature D, Higgins MC, Suermondt HJ, Young C, Owens DK. A randomized controlled trial of a computer-based physician workstation in an outpatient setting: implementa- tion barriers to outcome evaluation. J Am Med Inform Assoc 1996; 3:340-48.

6. Leiner F, Haux R. Systematic planning of clinical documentation. Meth Inform Med 1996; 35:25-34.

Address of the author:

Prof. J. Ozbolt,

Division of Clinical Informatics,

School of Medicine, McLeod Hall,

University of Virginia,

Charlottesville, VA 22903-3395, USA

E-mail: ozbolt@virginia.edu 